\title{
The evaluation of reproduction type of Puccinia graminis f. sp. tritici population prevailing in West Siberia
}

\author{
E.M. Sergeeva ${ }^{1 *}$, E.S. Skolotneva ${ }^{1}$, V.N. Kelbin ${ }^{1}$, S.I. Konkova ${ }^{2}$, M.A. Nesterov ${ }^{1}$, \\ E.A. Salina ${ }^{1}$ \\ ${ }^{1}$ Institute of Cytology and Genetics SB RAS, Novosibirsk, Russia \\ ${ }^{2}$ Novosibirsk State University, Novosibirsk, Russia \\ *e-mail: sergeeva@bionet.nsc.ru
}

Key words: stem rust, bread wheat

Motivation and Aim: Stem rust is a fungal disease of grasses (including agronomically important bread wheat Triticum aestivum L.) caused by Puccinia graminis, resulting in a significant decrease in grain production. The species $P$. graminis subdivides into specialized forms (f. sp.) passed through the adaptation to the specific range of host plants. The sexual process is optional for the fungal life cycle but leads to novel combinations of virulent genes as well as determines high genetic variability. In the case of sexual reproduction, an alternative host plant barberry Berberis vulgaris is served. The aim of the study is to establish the reproduction type (sexual/asexual) of the West Siberian population of $P$. graminis $\mathrm{f}$ sp. tritici occured on wheat.

Methods and Algorithms: The samples of infected leaves of barberry and stems of grasses (Triticum aestivum, Elytrigia repens and Dactylis glomerata) were collected in Novosibirsk area during June-August in 2016 and 2017. DNA was extracted according to modified CTAB method [1]. First to confirm the identity to Puccinia species the 45S rDNA internal transcribed spacer (ITS) regions were amplified and sequenced from the samples with the rust-specific primers pair ITS1RustF10d/ITS1rustR3c [2]. Then the samples were analyzed by PCR using the set of 16 publicly available SSR markers developed for P. graminis.

Results: The ITS analysis confirmed the stem rust pathogen in all 15 samples. The SSRs analysis showed the differences between the amplification spectra of $P$. graminis from barberry and wheat. The SSRs patterns from barberry and other grasses were identical. This means the local barberry is alternative host not for $\mathrm{f}$. sp. tritici but for other special form of the pathogen. Thus, we suggest an asexual type of reproduction for wheat stem rust population prevailing in West Siberia.

Conclusion: Since asexual reproduction type was established for P. graminis f. sp. tritici population prevailing in West Siberia, the source of inoculum in the region was considered as exogenous. The virulence structure of asexual wheat stem rust population migrating over West Siberia as well as determining the source of inoculum require further study. Acknowledgements: Supported by RFBR (No. 17-29-08018).

\section{References}

1. Justesen F. et al. (2002) The recent history of Puccinia striiformis f.sp. tritici in Denmark as revealed by disease incidence and AFLP markers. Plant Pathology. 51:13-23.

2. Barnes C.W., Szabo L.J. (2007) Detection and identification of four common rust pathogens of cereals and grasses using real-time polymerase chain reaction. Phytopathology. 97:717-727. 